\title{
Nanoparticles for Cytosolic Delivery of Important Biomolecular Drugs such as DNA, RNA, Peptides, and Proteins
}

\author{
Marián Sedlák*a, Čestmír Koňák ${ }^{\mathrm{b}}$ and Jiří Dybal ${ }^{\mathrm{b}}$ \\ ${ }^{a}$ Institute of Experimental Physics, Slovak Academy of Sciences, 04001 Košice, Slovakia \\ ${ }^{b}$ Institute of Macromolecular Chemistry, Czech Academy of Sciences, 16206 Prague, Czech Republic
}

\begin{abstract}
Taking advantage of the $\mathrm{pH}$ difference between an endosome and the cytosol (that of the endosome being lower by 1-2 units), it was proved that totally synthetic (exhibiting minimal antigenicity) pH-sensitive, membranedisrupting polymers such as poly(ethylacrylic acid) and poly(propylacrylic acid) significantly enhance in vivo transfections [1-3]. Their effect is based on the ability to disrupt membranes at the $\mathrm{pH}$ of the endosome but be non-lytic at $\mathrm{pH}$ 7.4. We have developed recently a new route to the preparation of poly(ethylacrylic acid) and poly(propylacrylic acid) nanoparticles based on the self-assembly mechanism of polymeric chains, i.e. via action of physical bonds [4,5]. Ideas behind this new route are reviewed together with the technical realization. Possible applications in drug delivery are discussed. We anticipate that these nanoparticles will also enable to utilize the EPR (Enhanced Permeability and Retention) effect in tumor tissues, meaning that certain sizes of large particles tend to accumulate in tumor tissue much more than they do in normal tissues. Nanoparticles have well controllable size, posses a mild acceptable degree of polydispersity, are stable over long periods of time (excellent shelf-life) and are stable in a broad range of salt concentrations including physiological. Nanoparticles have low density (loose and open internal structure) which is advantageous for loading with drugs. The developed self-assembly mechanism [4] is promising also with respect to the preparation of nanoparticles from other similar polymers.
\end{abstract}

Keywords: Cytosolic delivery, nanoparticle carriers, poly(ethylacrylic acid).

\section{INTRODUCTION}

One of the challenges in the drug delivery area is the intracellular delivery via endocytosis followed by endosomal escape due to the disruption of the endosomal membrane and release of important biomolecular drugs such as DNA, RNA, peptides, and proteins to the cytoplasm before they are trafficked to lysosomes and degraded by lysosomal enzymes. Totally synthetic (exhibiting minimal antigenicity) $\mathrm{pH}$ sensitive polymers such as poly(ethylacrylic acid) (PEA) and especially poly(propylacrylic acid) (PPA) significantly enhance in vivo transfections [1,2]. For instance, transfection of NIH3T3 fibroblasts with ternary mixtures of the cationic lipid carrier DOTAB, $\mathrm{pCMVb}$ plasmid DNA, with or without PPA, were compared [1]. It was shown that transfections were significantly higher with the addition of PPA. The difference was the more significant the higher the serum concentration was and the lower the charge ratio was [1]. Calcein delivery to cytosol via disruption of endosomes was monitored directly by confocal microscopy [3]. Cells were incubated with the membrane-impermeable fluorophore calcein with or without polymers added to solution. Three different polymers were used as well as a blank control: poly(methacrylic acid) (PMA), poly(ethylacrylic acid) (PEA), and poly(propylacrylic acid) (PPA) [3]. Cytosolic calcein fluorescence was increasing during several hours, the increase being the higher the more hydrophobic the polymer

*Address correspondence to this author at the Institute of Experimental Physics, Slovak Academy of Sciences, 04001 Košice, Slovakia; Tel/Fax: +421 55 7922245; E-mail: marsed@saske.sk was (PPA > PEA > PMA). A new approach to the preparation of poly(ethylacrylic acid) and poly(propylacrylic acid) nanoparticles based on the self-assembly mechanism of polymeric chains was recently developed in our laboratory $[4,5]$. We expect that the utilization of the EPR (Enhanced Permeability and Retention) effect in tumor tissues via these particles is possible, too.

\section{EXPERIMENTAL}

\section{Material}

Poly(ethylacrylic acid), $M_{w}=18600, M_{n}=12400$, $M_{w} / M_{n}=1.5$, and poly(propylacrylic acid), $M_{w}=47000$, $M_{n}=22400, M_{w} / M_{n}=2.1$, were obtained from Polymer Source, Canada, and used as delivered.

\section{Light Scattering}

The static light scattering (SLS) as well as dynamic light scattering (DLS) measurements were made using a Stabilite 2017-04S argon laser (Spectra Physics, Mountain View, CA) with $514.5 \mathrm{~nm}$ vertically polarized beam. Laser power was limited to $100 \mathrm{~mW}$ maximum via neutral density filters. A laboratory made goniometer with angular range from $30^{\circ}$ to $150^{\circ}$ was used to collect data for both static and dynamic light scattering experiments. The scattering cell was thermostated at $25^{\circ} \mathrm{C}$ with a precision of $\pm 0.1^{\circ} \mathrm{C}$. An ALV5000/E correlator with a fast correlation board option (ALV, Langen, Germany) was used for photon correlation measurements. Characteristic decay times of dynamic modes and their relative amplitudes were evaluated through the moments of distribution functions of decay times obtained 
by fitting correlation curves using CONTIN [6] and GENDIST [7,8] programs.

\section{Differential Refractometry}

Refractive index increment $d n / \mathrm{dc}$ was measured via DNDC-2010 differential refractometer from WGE Dr. Bures (Germany). The $535 \mathrm{~nm}$ wavelength was used. Since light scattering was measured at $514.5 \mathrm{~nm}$, the error was negligible. The typical noise level was very small, less than 5 X $10^{-9}$ RIU.

\section{Electrophoretic Light Scattering}

Zeta potentials $(\zeta)$ were measured using a Nano-ZS, Model ZEN3600 (Malvern Instruments, UK) equipped with a He-Ne laser operating at a wavelength of $632.8 \mathrm{~nm}$. The zeta potential was measured at least ten times to check for repeatability. The measured electrophoretic mobilities $[\mu \mathrm{m}$ $\mathrm{cm}(\mathrm{Vs})^{-1}$ ] were converted to zeta potential $(\mathrm{mV})$ using the Smoluchowski approximation.

\section{Differential Scanning Calorimetry (DSC)}

Measurements were performed on the Perkin-Elmer Pyris 1 DSC calorimeter. Samples of about $5 \mathrm{mg}$ were closed in Al sample pans and the system was flushed with dry helium during the DSC scan. The temperature scale was calibrated according to the melting points of cyclohexane and indium. The power output scale was calibrated with indium.

\section{FTIR Spectroscopy}

ATR FTIR spectra were collected on a Nicolet Nexus 870 FTIR spectrometer purged with dry air and equipped with a cooled mercury-cadmium-telluride (MCT) detector. Samples were measured on a horizontal micro-ATR Golden Gate unit (SPECAC) with a diamond prism; spectral resolution was $4 \mathrm{~cm}-1$. All the spectra were processed by ATR correction.

\section{RESULTS AND DISCUSSION}

The self-assembly process was designed based on the following idea. A thermosensitive polymer solution is heated, the worsening of the solvent quality [9] upon increasing temperature forces polymer chains to associate. At a close-enough distance, hydrogen bonds between chains become also operable and contribute to the stabilization of nanoparticles. As a result, nanoparticles do not dissolve upon cooling (hydrogen bonds strengthen upon cooling) and remain stable. Due to the charge on nanoparticles, these are resistant to agglomeration during the heating process as well as after cooling down to ambient temperature. Fig. (1) shows an example of the preparation process as monitored on-line by light scattering.

A detailed and exact characterization of nanoparticles was done after the preparation process on highly diluted samples (Table 1). Particles are relatively loose (low density) which may be advantageous for loading and release of low molecular weight compounds. They have a moderate and acceptable polydispersity. An excellent long-term stability (excellent shelf-life) of the particles was found by measurements repeated after more than one year from the particles preparation.
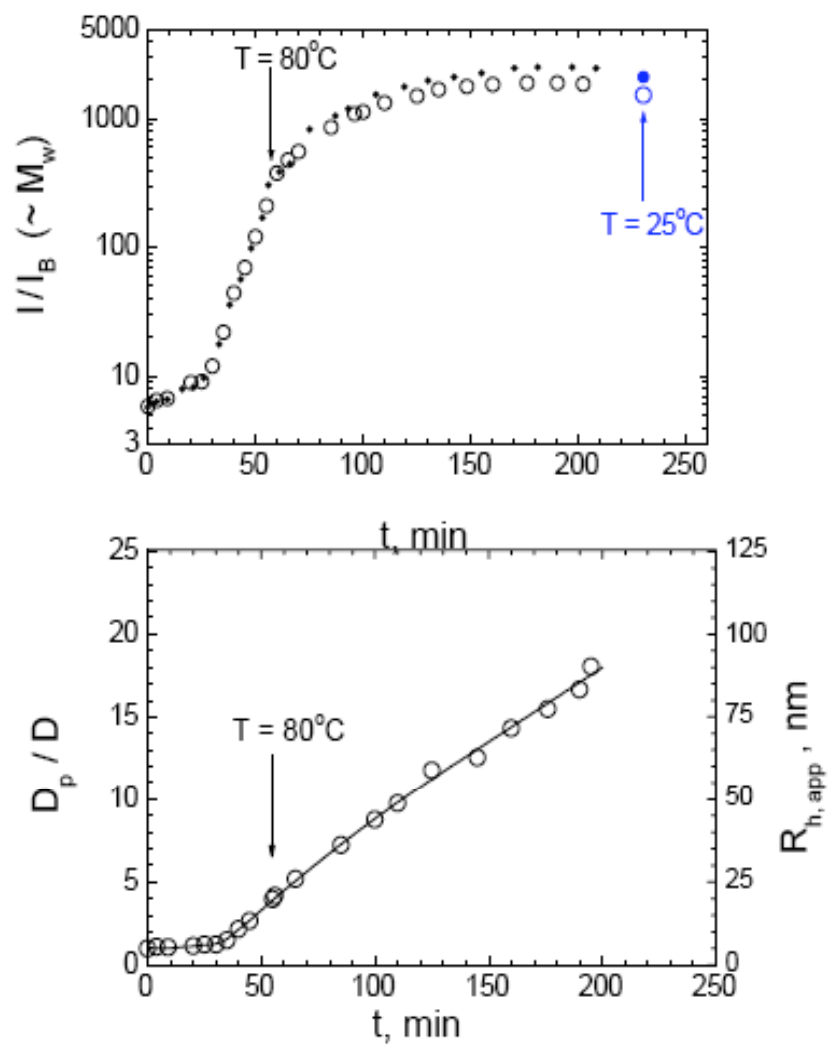

Fig. (1). On-line monitoring of the growth of polymeric nanoparticles by light scattering. Scattering intensity $I$ grows due to the increase of the molecular weight $M_{\mathrm{W}}$ of the particles. Increase of $D_{p} / D$ is due to the increase of the apparent hydrodynamic radius $R_{h \text {,app }}$ of nanoparticles. $D$ is the measured diffusion coefficient, $D_{p}$ is the diffusion coefficient expected at temperature $T$ provided that no change occurs in the sample upon heating except changes of $T$ and solution viscosity $\eta$. PEA solution, $\alpha=0.21, c=16 \mathrm{~g} / \mathrm{kg}, c_{S}=$ $127 \mathrm{mM} \mathrm{NaCl}$. Solution was heated from $T=25^{\circ} \mathrm{C}$ to $T=80^{\circ} \mathrm{C}$ at the heating rate $1^{\circ} \mathrm{C} / \mathrm{min}$. Scattering angles $90^{\circ}(\mathrm{O})$ and $45^{\circ}(\bullet)$, respectively. Scattering after cooling down to $T=25^{\circ} \mathrm{C}$ is also shown at angles $90^{\circ}(\mathrm{O})$ and $45^{\circ}(\bullet)$, respectively. Cooling down was realized simply by withdrawing the sample from the $80^{\circ} \mathrm{C}$ bath. (From Sedlák, M.; Koňák, Č.; Macromolecules, 2009, 42, 74307438).

The parameters of nanoparticles as shown in Table $\mathbf{1}$ can be controled mostly by the heating treatment, especially by the time of heating. The key physico-chemical parameter which controls the self-assembly process is the degree of neutralization [10] of the polymer $\alpha$ (Fig. (2)). This degree controls a delicate balance between hydrophobic and electrostatic interactions which act in an opposite direction.

Fig. (3) shows calorimetric data, namely results from a measurement in the standard DSC mode with a constant heating rate $1{ }^{\circ} \mathrm{C} / \mathrm{min}$ in heating and cooling runs. The most typical sample used in our work ( $c=16 \mathrm{~g} / \mathrm{kg}, \alpha=0.21$, Fig. (1)) was scanned in the temperature interval from 21 to 81 ${ }^{\circ} \mathrm{C}$. Two distinct features are found: (i) the transition is oneway (fully irreversible) and (ii) the transition is rather broad starting around $T=40^{\circ} \mathrm{C}$ and reaching maximum between $50^{\circ} \mathrm{C}-70^{\circ} \mathrm{C}$. Calorimetric data thus confirm main results from light scattering concerning the irreversibility and a 
Table 1. Parameters of Nanoparticles Created by the Procedure Shown in Fig. (1)

\begin{tabular}{|l|}
\hline Hydrodynamic radius $R_{h}=85.6 \mathrm{~nm}$ \\
\hline Radius of gyration $R_{g}=81 \mathrm{~nm}$ \\
\hline Ratio of the radius of gyration and the hydrodynamic radius $R_{g} / R_{h}=0.95 \pm 0.1$ \\
\hline Size polydispersity PDI $=(\sigma /<R>)^{2}=0.18$ \\
\hline Molecular weight $M_{w}=3.92 \times 10^{7} \mathrm{~g} / \mathrm{mol} \quad(\mathrm{dn} / \mathrm{dc}=0.202 \mathrm{~mL} / \mathrm{g})$ \\
\hline Density $\rho=3 M_{w} / 4 \pi N_{A} R_{h}{ }^{3}=0.025 \mathrm{~g} / \mathrm{mL}$ \\
\hline Zeta potential $\zeta=-35 \mathrm{mV}$ \\
\hline
\end{tabular}

broad (slow) transition upon heating. which support light scattering results.
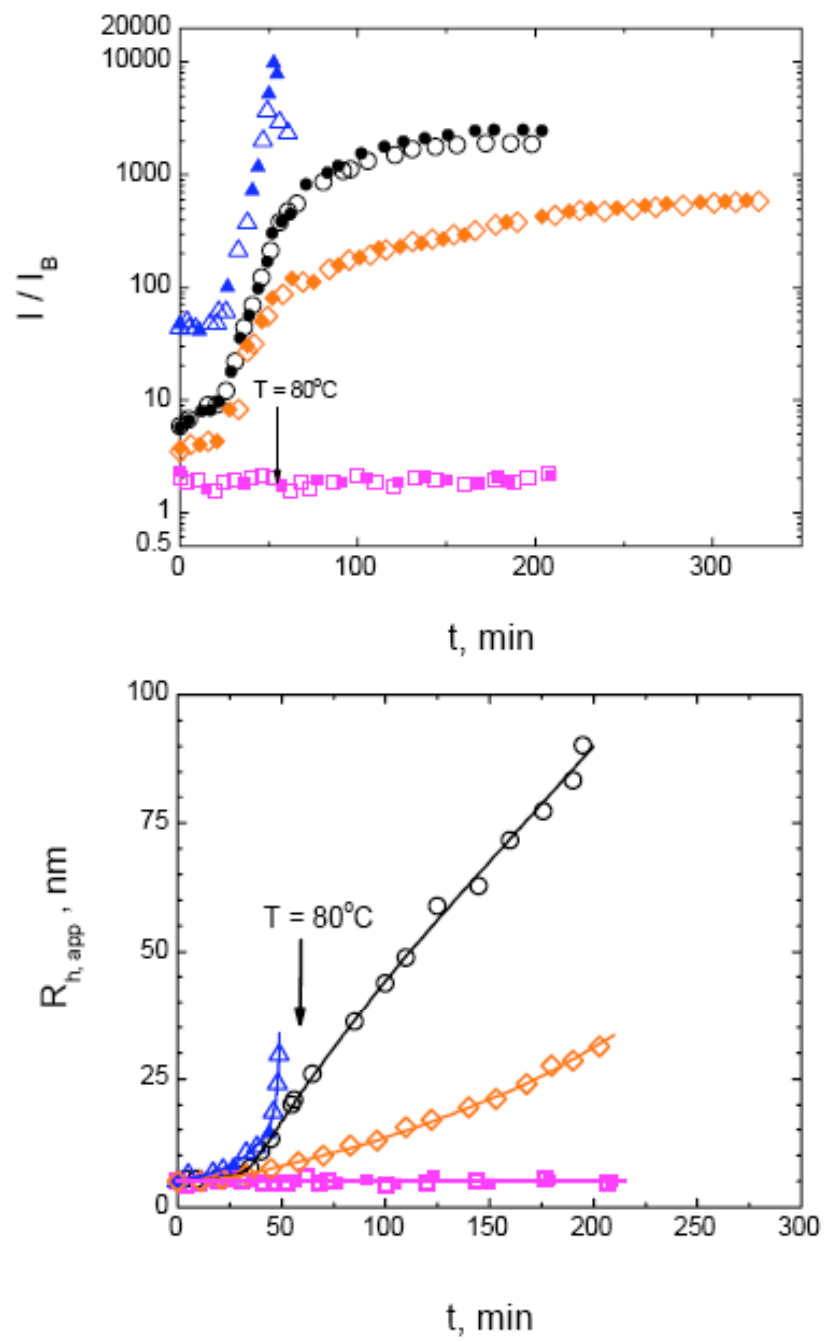

Fig. (2). Effect of the degree of neutralization $\alpha$ on the selfassembly behavior of PEA upon heating. Polymer concentration was $c=16 \mathrm{~g} / \mathrm{kg}$. Solutions were heated from $T=25^{\circ} \mathrm{C}$ to $T=80^{\circ} \mathrm{C}$ at the heating rate $1^{\circ} \mathrm{C} / \mathrm{min}$ and then kept at $T=80^{\circ} \mathrm{C}$. Scattering angles $90^{\circ}$ (open symbols) and $45^{\circ}$ (closed symbols), respectively. $\alpha=0.150(\Delta), 0.214(\bigcirc), 0.250(\diamond)$, and $0.319(\square)$. Scattering intensity $I$ grows due to the increase of the molecular weight $M_{\mathrm{W}}$ of the particles. $R_{h, a p p}$ is apparent hydrodynamic radius of nanoparticles. (From Sedlák, M.; Koňák, Č.; Dybal, J.; Macromolecules, 2009, 42, 7439-7446).

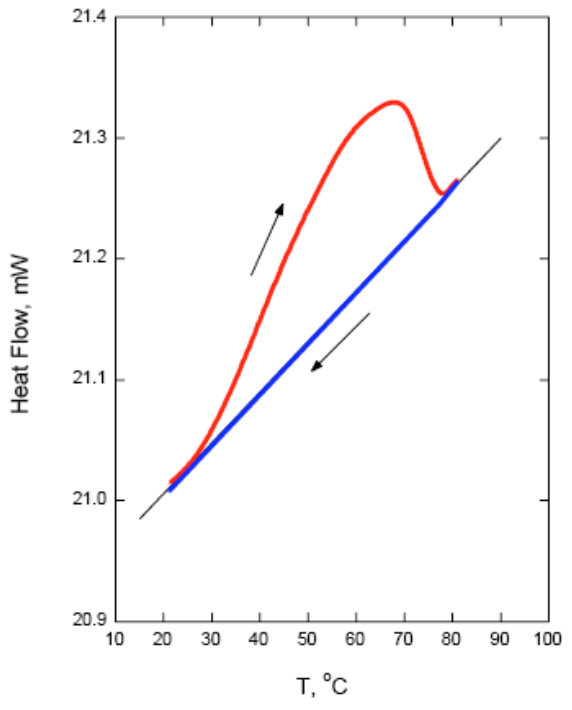

Fig. (3). Differential scanning calorimetry (DSC) data on a PEA solution, $\alpha=0.21, c=16 \mathrm{~g} / \mathrm{kg}, c_{s}=127 \mathrm{mM} \mathrm{NaCl}$. Measurements in the standard DSC mode were performed with a constant heating rate $1{ }^{\circ} \mathrm{C} / \mathrm{min}$ in the heating and cooling runs. (From Sedlák, M.; Koňák, Č.; Dybal, J.; Macromolecules, 2009, 42, 7439-7446).

In order to elucidate forces behind the irreversibility of the process of nanoparticle formation we carried out FTIR measurements. In order to avoid a strong contribution of $\mathrm{H}_{2} \mathrm{O}$ to IR spectra, all measurements were done in $\mathrm{D}_{2} \mathrm{O}$ solutions. This substitution has no effect on the PEA selfassembly as checked by separate light scattering measurements. FTIR spectra shown in Fig. (4) were measured on the most typical solution used in these studies, which was selected as optimum for the nanoparticle formation, i.e. PEA in $0.12 \mathrm{M} \mathrm{NaCl}, \alpha=0.21, c=16 \mathrm{~g} / \mathrm{kg}$. Spectra were accumulated prior to heating and after heating according to the usual heating scheme from Fig. (1). For comparison, solution with the degree of neutralization $\alpha=$ 1.0 (fully neutralized polymer) was also measured. The appearance of the doublet at 1752 and $1650 \mathrm{~cm}^{-1}$ is connected with the emergence of hydrogen bonds $\mathrm{COOH} . . . \mathrm{COO}^{-}$and the doublet is assigned to the strongly coupled stretching vibrations of the carbonyls in the $\mathrm{COOH}$ and $\mathrm{COO}^{-}$groups. The assignment is supported by DFT calculations of vibrational frequencies in the hydrogen bonded structures: 1747 and $1681 \mathrm{~cm}^{-1}$. FTIR measurements suggest that the main stabilization forces responsible for the irreversibility of the self-assembly are hydrogen bonds, especially $\mathrm{COOH} . . . \mathrm{COO}^{-}$bonds, which have significantly 
higher interaction energies compared to $\mathrm{COOH}$...COOH bonds.

Nanoparticles are stabilized by hydrogen bonds and presumably also by frozen-in hydrophobic interactions between polymer molecules. This reminds the stabilization of globular proteins. These are known to be denatured by means of urea addition. In order to test our conclusions about the stabilization forces, urea was added to solutions of PEA nanoparticles. While no effect was seen at low urea concentration (up to $\sim 300 \mathrm{mM}$ ) a very clear process of particle disintegration ("denaturation") can be monitored in real time at higher urea concentrations $(\sim 1 \mathrm{M})$.

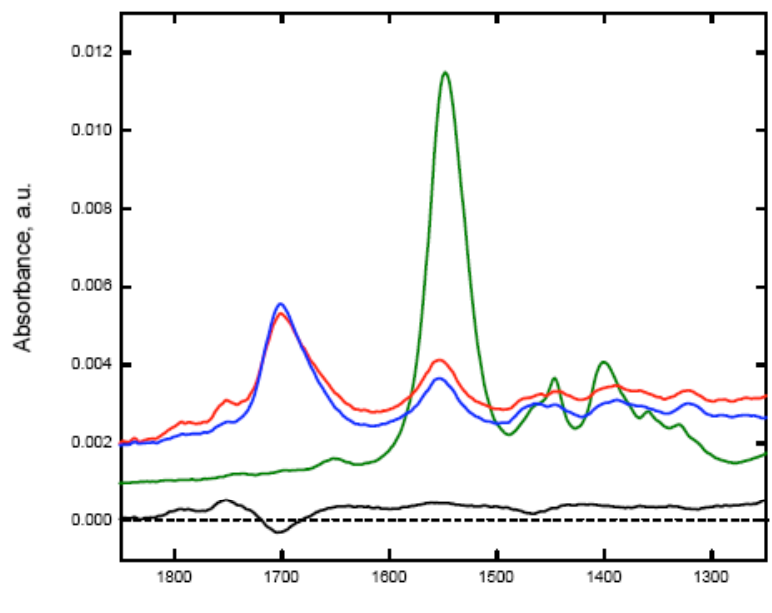

Wavenumber, $\mathrm{cm}^{-1}$

Fig. (4). FTIR spectra. PEA, $\alpha=0.21, c=16 \mathrm{~g} / \mathrm{kg}, c_{s}=127 \mathrm{mM}$ $\mathrm{NaCl}$ prior to heating (- - $)$ and after heating according to the usual heating scheme from Fig. (1) (- - ). PEA, $\alpha=1.0, c=20$ $\mathrm{g} / \mathrm{kg}$ ( - ). Differential spectrum ( - - $)$ : spectrum after heating - spectrum before heating. All spectra were acquired in $\mathrm{D}_{2} \mathrm{O}$ solutions. Spectrum of $\mathrm{D}_{2} \mathrm{O}$ was subtracted. (From Sedlák, M.; Koňák, Č.; Dybal, J.; Macromolecules, 2009, 42, 7439-7446).

Nanoparticles were also tested with respect to the stability in saline solutions of various concentrations and found to be stable from deionized water up to $0.6 \mathrm{M} \mathrm{NaCl}$, i.e. including physiological salt concentration $(0.15 \mathrm{M} \mathrm{NaCl})$. Nanoparticles were succcesfully prepared also from poly(propylacrylic acid). This work is currently in progress and suggests that our new approach to the nanoparticles formulation may be applicable to a wider range of polymers. Poly(propylacrylic acid) also showed a stronger ability to disrupt endosomal membranes. The self-assembly mechanism is schematically shown in Fig. (5) together with calculated model structures showing carboxylic-carboxylate hydrogen bonds.

\section{ACKNOWLEDGEMENTS}

Support from the Slovak grant agency VEGA (grant No. $2 / 0215 / 10)$ is acknowledged. This work was realized within
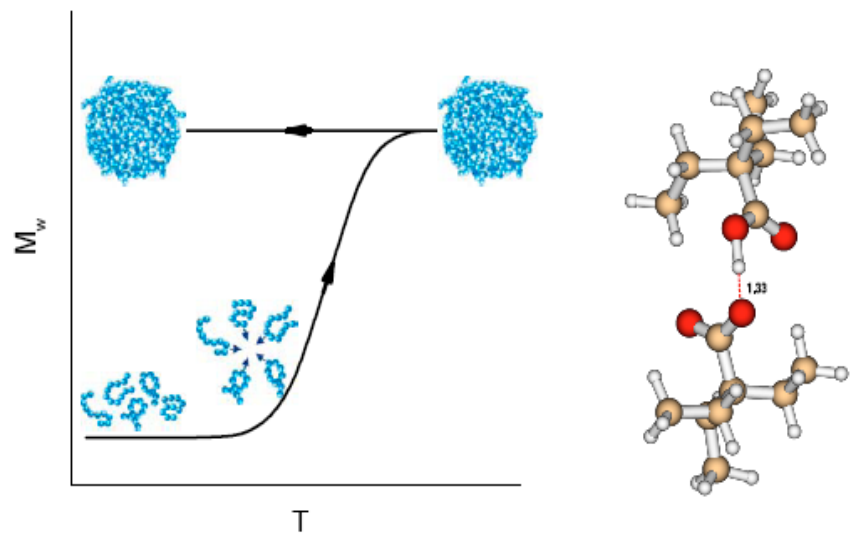

Fig. (5). Schematic diagram of the self-assembly mechanism (left). Optimized geometries of the model structures showing carboxyliccarboxylate hydrogen bonds (right). Calculated by quantum mechanical calculations at the B3LYP/6-31G(d) level, distance in Å. (From Sedlák, M.; Koňák, Č.; Dybal, J.; Macromolecules, 2009, 42, 7439-7446).

the frame of the project "Centre of Excellence for Advanced Materials with Nano- and Submicron Structure", which is supported by the Operational Program "Research and Development" financed through the European Regional Development Fund.

\section{REFERENCES}

[1] Kyriakides, T.R.; Cheung, C.Y.; Murthy, N.; Bornstein, P.; Stayton, P.S.; Hoffman, A.S. pH-sensitive polymers that enhance intracellular drug delivery in vivo. J. Control Release., 2002, 78, 295-303.

[2] Hoffman, A.S.; Stayton, P.S.; Press, O.; Murthy, N.; Lackey, C.A.; Cheung, C.; Black, F.; Campbell, J.; Fausto, N.; Kyriakides, T.R.; Bornstein, P. Design of "smart" polymers that can direct intracellular drug delivery. Polym. Adv. Technol., 2002, 13, $992-$ 999.

[3] Hoffman, A.S.; Jones, R.A.; Cheung, C.Y.; Black, F.E.; Zia, J.K.; Stayton, P.S.; Wilson, M.R. Poly(2-alkylacrylic acid) polymers deliver molecules to the cytosol by $\mathrm{pH}$-sensitive disruption of endosomal vesicles. Biochem. J., 2003, 372, 65-75.

[4] Sedlák, M.; Koňák, Č. A new approach to polymer self-assembly into stable nanoparticles: poly(ethylacrylic acid) homopolymers. Macromolecules, 2009, 42, 7430-7438.

[5] Sedlák, M.; Koňák, Č.; Dybal, J. Heat-set poly(ethylacrylic acid) nanoparticles: combined light scattering, calorimetric, and FTIR study. Macromolecules, 2009, 42, 7439-7446.

[6] Provencher, S. W. CONTIN: a general purpose constrained regularization program. Comput. Phys. Commun., 1982, 27, 213227.

[7] Jakeš, J. REPES method for fitting dynamic light scattering data. Czech. J. Phys., 1988, 38, 1305-1315.

[8] Štěpánek, P. In Dynamic Light Scattering. The Method and Some Applications; Brown, W., Ed., Clarendon: Oxford, 1993, pp. 177240.

[9] Solvent quality means the ability of solvent to solvate the polymer. The better the solvent quality the more repulsive is the interaction between polymers. The worse the solvent quality the more attractive is the interaction between polymers.

[10] Degree of neutralization is defined as a ratio of molar concentrations of added hydroxide and monomer, respectively. It is roughly equal to the degree of ionization. 